\title{
Data Fusion for the Management of Multimedia Documents
}

\author{
Françoise Deloule \\ Équipe Condillac - LISTIC \\ Université de Savoie \\ Bât. Mont Blanc \\ 73376 le Bourget du Lac Cedex. \\ France \\ francoise.deloule@univ-savoie.fr
}

\author{
Daniel Beauchêne (Équipe Condillac), \\ PatrickLambert (Équipe TI), \\ LISTIC - Université de Savoie \\ France \\ daniel.beauchene@univ-savoie.fr \\ patrick.lambert@univ-savoie.fr \\ Bogdan Ionescu \\ University "Politehnica" Bucharest, \\ LAPI, 061071, Bucharest, Romania \\ BIonescu@alpha.imag.pub.ro
}

\begin{abstract}
Multimedia documents are increasingly numerous. Their efficient management requires tools to provide services that measure up to users' expectations, based on the contents of these voluminous document databases. This implies a number of challenges. Although we can extract highly symbolic concepts from texts, a wide semantic gap appears when processing images and sound. Thus, we propose using data fusion to capture the semantic field of the elements extracted from these media. On the other hand, if creating ontology makes it possible to manage the concept of "trade" in texts, additional data can be exploited from document contents. In the first instance, we suggest bringing together concepts and words through data fusion.
\end{abstract}

Keywords: fusion of heterogeneous data, semantic fields, ontology, terminology.

\section{Introduction}

In our contemporary context, we are consistently confronted by prolific fields of information, and multimedia documents are being produced at a breathtaking pace. Their management and exploitation trigger challenges for operations that exploit the contents of such documents, without being limited to the sole use of arbitrary keywords in order to satisfy users' needs. Each medium has its own modes of expression and the accessible inter and intra media semantic levels vary significantly. Low semantic numeric indicators are available for images and sound whereas concepts with strong semantic value can be extracted from text-based data. However, knowledge extraction and concept construction are no easy matters. They often require the fusion of highly heterogeneous data, in term of semantic granularity, context and interpretation, quality and reliability, for instance.

Using a specific application (section 2) coupled with the essential assistance of experts in the field, we propose reducing the current semantic gap between data stemming from different media (section 3) to model and exploit data from different semantic fields (section 4) before highlighting the complementary approaches (section 5). Various information fusion approaches will be used to meet these challenges. Finally, the avenues to be explored through our future work will be presented.

\section{A support application}

In order to present a concrete illustration, a knowledge management issue in the field of animated cinema will be investigated. Indeed, every year, the CICA (a French acronym for "International Center of Animated Cinema at Annecy" [1]) is faced with the double dilemma of managing FIFA's (French acronym for "International Festival of Animated Film") new releases as well as managing, over time, the legacy left to testify to the evolution of this art. These two facets are relevant for various users: cinema teachers and students, projection organizers, professionals, the discriminating public or amateurs.

A wide variety of data is collected. Obviously, there are the films themselves, kept and preserved by the CICA on various media and currently being transferred onto a numeric support, in order to meet the twin objectives of preserving them in good condition over time, while also rendering them available to users. There is also an important database that contains text documents pertaining to the films, such as the festival registration cards and technical and descriptive sheets for a certain number of films. CICA also owns various generic 
documents pertaining to animated cinema: specialized dictionaries, books covering various topics, biographies, newspaper clippings, critiques, advertisements, pictures, specialized magazines, documentary files, etc. CICA is also concerned with exploiting, broadcasting and recognizing the knowledge and competencies of its experts who, in turn, expect a powerful film analysis and characterization platform. Cica has already more than 25.000 films in store, references nearly 55000 professionals, and grows rich by approximately 1500 films each year at the time of the festival. Experts helped us to select a representative extract of this corpus of films and texts and they could validate some of our proposals. Initially, we restricted our field of study to the contents of the descriptive card-index of each film. They describe films by the title of the artistic work (in original language, French and English), the nationality and the year of production, names of the different "authors": scenarist, graphics, sound, etc... indications on the techniques, the duration, the support, the kind of film, the age of the public concerned, the category of competition chosen for this festival, a synopsis in English and French. These data are complete enough for the films which were presented at a festival, much less for the others. The quality of the data is very heterogeneous for various reasons: all are not provided by the authors of works, some remain absent, others "are improvised" in order to seize the data absent in the base, some are obtained by translations whose quality varies according to the knowledge of this domain by the translator, others are provided by the authors who interpret the request differently... For example, the text of the field "synopsis" can according to cases contain a summary of film, an eye-catching advertisement or a criticism.

\section{Non-text Media}

Using this corpus of texts and films, we propose powerful knowledge and management tools. Although current knowledge and data processing tools make it possible to extract strong semantic concepts out of texts, such is not the case for sound and picture processing. Nevertheless, they cannot simply be ignored. A minimum of two components can be analyzed on such media: utterances (written into the shots or heard on the soundtrack) that can be transcribed verbatim as well as all the other sound and picture components. Here, we deliberately ignore the utterances which trigger difficulties such as those pertaining to the texts, as described further on.

What else can be extracted from a soundtrack or a set of consecutive shots? In the case of films, a large amount of information can be perceived and analyzed. A sophisticated assembly of images and sounds is designed to capture the attention and interest of viewers, to stimulate their sensory capacities and trigger emotions and reactions. Feelings thus constitute other relevant ingredients that can be observed in the framework of a picture and sound investigation. For instance, we are looking to discover the importance of the colours selected to paint a warm or grim atmosphere. Then, observations will be carried out to assess whether such data and film genre correlate.

However, to extract this information from pictures, the main indexing techniques available are those developed for still-frame processing. Unfortunately, such techniques are inappropriate for video stream processing due to movement recognition and the humongous quantity of data to be processed. Recent video stream studies carried out by our team [2] propose to define "atmosphere"" indicators, through the exploitation of the colours used and their distribution as well as the rhythm of shot substitution and the detection of certain special effects. To do so, fuzzy logic based data fusion will be used. Correlations can then be made between these indicators and the textual components (in part 5).

The indicators built starting from the numerical data resulting from these analyses enable us to build indicators with stronger semantic contents [2], [3]. By example on our corpus, we built a descriptor of "environment" for the animation movies, starting from three components which are the rhythm, colour and special effects. Each component is built starting from indicators. The rhythm is the result of a fusion of the indicators' speed, activity and explosiveness. These indicators of activity and speed are the result of numerical data relating to the changes of plans (number, frequency and length, compared to the total duration of film). Explosiveness is the interpretation, for the animated cinema, of the specific colour effect named "short colour change". For the study of the component "colour", we use initially a process of reduction of colours using the Floyd-Steinberg error diffusion filter, then a study of the distribution of the colours based on the Itten's colour wheel [4]. Thus, we determine the dominant tonality, by the fusion of the results of calculations of diversity and variety of the colours used, of the colour contrast, warmth, the intensity and saturation of colours. These indicators, as for the rhythm, are obtained by application of fuzzy logic to the fusion of the numerical results. The special effects concern on the one hand a technique of changes of plans like the dissolve, involving in animated films an impression of mystery, on the other hand the effects related to the texture of the image studied on representative fixed images (images of summary). For example, the handling of powder under the camera, a specific technique of animation movies, returns a particular texture. Environment is thus a descriptor whose semantic field is increased. It is the interpretation of the result of fusions of indicators of low semantic level resulting from the analyses of images.

These descriptors will facilitate the establishment of correlations between the picture and text data, information or knowledge that pertains to these multimedia objects. Our sound experiments will seek to extract the same type of knowledge (sound effects, music, rhythm and beat, detection of sharp sounds, etc.). 


\section{Texts}

A plethora of text data is available. Their diverse origins, contexts, usage and mode of creation... hinder automatic semantic-based processing Text that is external to the films or multimedia objects will be favoured. Indeed, we postulate that the data pertaining to the text included in these multimedia objects (written into the shots or heard on the soundtrack) will be interpreted, modeled and exploited in the same way as the external text (such as synopsis, critiques, summaries, reviews, showings, book contents), as soon as transcription techniques designed to extract written texts, are available. Paratext will thus be investigated. Paratext refers to records used to describe, review or characterize films through an external approach. More specifically, the information contained in the film description database (festival registration cards) will be investigated.

These texts were written with at least two levels of language [5]: on the one hand, technical words which are specific to the field of animated cinema, for which a trade terminology and trade ontology can be established. Not only do terminology and ontology make it possible for field specialists to communicate and share their knowledge in an efficient, reliable and clear manner, but they also make it possible for novice users to become acquainted with the field in a simple, guided, relevant and user-friendly way.

In addition, there are texts which contain a more extensive vocabulary composed of not only trade terms, but also the words which make up speakers' statements (for instance, a journalistic expression used in a film review, generic terms found in scientific journals) as well as the colourful vocabulary of the language, the style and expressions used, to summarize the story of a movie or write a synopsis for example. In the remainder of this paper, such texts will be referred to as "free text".

These two sets of vocabulary will be addressed consecutively. First, the "technical" data and texts will be used to create a trade ontology and terminology. Then, the free texts will be investigated. Finally, we will determine if the knowledge extracted from these texts can be combined with the previously modeled knowledge, or whether it is best to build new categories within, or beside, the ontology.

\subsection{Concepts and Trade Terms}

To ensure that textual data is processed in an efficient and relevant manner, we seek to make concepts and their relationships emerge beyond the words, in order to model and formalize the knowledge conveyed by the media. Without reiterating definitions or the utility and relevance of ontology, we know that they cannot be universal. To be exploitable, an ontology [6] must be related to a single field or a trade and the concepts thus organized are defined for this particular context. An ontological terminology approach was adopted [7]. This approach favours the creation of complementary trade ontology and terminology, it facilitates the extraction and organization of deep knowledge from the field and it allows for the removal of ambiguities found in natural language. The model selected, called OK [8], rests upon principles resulting from several disciplines such as epistemology (defining the concept with their essential characteristics and not only with their describing properties), linguistics (the sign is arbitrary; language consists of a system used to structure words), artificial intelligence (the impact of the language representation on modeling) and logic (semantical logic and set-theoretic knowledge, $a$ posteriori formalization of knowledge, concepts and their relationships).

This ontology is built upon the following principles and definitions: a field is described by several categories which can then be combined for various system uses. For example, in animated cinema, techniques, genres and target audience consist of various distinct categories. A category associates the terms whose definitions are semantically linked. For example under the category "technique", expressions such as "Plasticine", "shadow figures" or "celluloid drawings" are found.

Terms are gathered in each category according to their nature, which makes it possible to define concepts, differences and attributes, while capturing certain conceptual relationships. Concepts refer to knowledge that pertains to a certain plurality of elements rather than a given singular unit of knowledge. Each concept within a category is defined according to the preceding one in terms of "kind-of", a subsumption relation (for example, in the category "technique", "shadow figure" is a "kind of" "cut-out") and a "complement" indicates what differentiates it from the previous element. This "complement" constitutes the most basic significant element from which concepts are built and shared, based upon the very nature of the object. Consequently, a concept either has or does not have one essential characteristic, and takes its place within a tree structure, within which logic elements can be applied (a posteriori formalization, logical semantics and set-theoretic knowledge). In this way, the lighting mode differentiates "shadow figures" from "cut-out elements". In the end, the attribute makes it possible to describe the state of the concept rather than its very nature (for instance, the material used to create "shadow figures" consists of an attribute). To organize the concepts, a "grid of differences" can be built by considering the essential characteristics of the concepts: for each given concept, we state whether a characteristic includes or belongs to a certain sense. Successive analyses and reassemblies make it possible to define and organize concepts. We build an ontology on a subjacent form of "Porphyry's tree", a permutable binary tree composed of levels which ensures the system's sturdiness and validity over time and use. We supplement the relevant terminology with new term used for describing some intermediate concept or new specific differences. We thus paint a clear picture of the role(s) played by each term within a given trade. This ontology, 
that supports the conceptualization of the field elements, is submitted to experts for validation purposes. In the case of the study carried out at the CICA, it was necessary to return to the databases and, on occasion, to the films themselves in order to select the terminology that defines the concepts and their synonyms. Such a task was also carried out in order to establish a consensus.

Finally, the ontology must be validated on the entire corpus. Corpus elements which do not seem to fit into any category must be examined to assess the necessity of adding a new concept and its associated specific differences or whether this concept was actually identified by a synonym or a translation of this term. Trade ontology and terminology are mutually completed, validated and exploited. The ontology built for the animated films currently includes the following categories: GENRE, PRODUCTION'S TECHNIQUES, AUDIENCE and PROCESS. Incidentally, the task of defining and organizing concepts generated a new version of the registration form which had become too complex, ill-adapted and obsolete over time. The form now includes solely the elements used to enrich a knowledge base, those that pertain to the ontology.

\subsection{An Extensive Vocabulary}

Although the construction of such trade ontology requires strenuous efforts, this is only one important step to manage the text data that must be processed in the framework of this study. Contrary to the technical fields where document contents necessarily converge towards a given goal, the artistic fields are described with a much higher level of freedom on varied and open topics. Techniques, basic knowledge, skills and know-how are critical and they must be captured to be transferred to or to contribute to the value of the CICA heritage. However, it does not make it possible to exploit the creative value of each film, to translate the unique content of multimedia documents. Nevertheless, numerous applications need access to this content. Thus, texts that describe film contents (e.g., a summary or synopsis provided with the festival registration card) are used to identify a film theme, for example. This field is written in natural language [5]: the vocabulary is rich and original and writing styles vary. These documents contain realistic descriptions made up of metaphors or symbolic expressions. Such texts are brief: they only contain a few words or lines.

How can these words be exploited? What is the most effective manner to identify relevant concepts or semantic fields without obliterating the artists' creativity? This study provides a more complete approach which can't take place here. We seek to analyze a single section of the texts, which includes common elements with the technical vocabulary and the trade ontology and terminology. First of all, as texts are short and original, they render inefficient any occurrence counting technique or method used to discover a concept or a significant semantic element.
Then, a quick observation of these brief texts indicates that a certain number of words are related to the concepts found in the previously built ontology. This ratio will be studied by observing the location and role of the words in the summary, its semantic contribution to define the film contents or the context of this description. Indeed, we know that the word semantics are intimately linked to their usage contexts.

However, we believe that the context of writing, producing and using these summaries is mainly driven by the trade ontology and terminology. In addition, it is partly defined by the picture and sound indicators. Also, for the summaries or synopsis which lend themselves to it, an attempt will be made to identify all occurrences of trade terms (or their synonyms [9] which are likely to enrich the terminology and ontology). As soon as one of these terms occurs in the summary, a correlation graph is created: a central role is assigned to this term. Its position and relationship in the sentence and the entire text is analyzed. Various distances can be defined to study the relationship between words with this specific context: syntactic distance based on the grammatical structures of the sentence, lexical, vectorial or semantic distances between the words, each bringing, with an adapted weight, an element of meaning for approaching with ontological concepts. Our approach is inspired by semantic cartography work [10], as well as various studies carried out by way of analysing comments by consumers [11]. We are particularly interested in a qualitative examination of these relationships, not through a comprehensive study, but rather by seeking certain characteristic elements (e.g., is the sentence negative, affirmative or interrogative? Is the word used in a positive manner or tainted by a pejorative connotation? What is the relative distance between the words of the sentence/text and those that include the traits of a sought element?). This qualitative approach also makes it possible to calculate the resulting distance between words and ontological concepts. The estimated film characteristics can then be evaluated, matched or discarded. Indeed, these results are compared with brief descriptions of corresponding fields on a descriptive card, identified by matching one of the ontology categories. For example, if the summary includes a word that describes a technique, the technical field of the card is filled out. If two words correspond, a match is found: the ontology distance is calculated and, if significant, the cohesion or ambiguity issue is resolved.

For a clear illustration, in a very restrictive way, take a film whose registration card indicates "genre: dramatic and artistic" and the following summary: "A woman's ordeal: a young man returns after being absent several years. What is the relationship between the two actors? Why such a long separation? A three-act tragedy: wait, confront and accept". In this summary, the word "tragedy" can trigger the use of "drama" in the ontology genre category This relation is defined, on one hand, by the fact that the terms "drama" and "tragedy" can be 
considered synonyms in the ontology, but especially by the definition, obtained by considering specific successive differences of the word "tragedy" in the ontology. Hence, "tragedy" becomes a central term to exploit from the text. As far as the relationships between the other words in the text, are concerned, on the one hand, "wait" and "confront" appear in the same sentence and, on the other hand, "separation" and "absent" also appear in the text (only certain relationships are retained. For example, notice that the correlation between "separation" and "return" could give clues as to the dramatic aspect of the story). These words contribute to the consolidation of the genre identified as "drama". Moreover, since an "allusion" to the genre was detected and identified, the terms used in the field genre on the registration card become particularly relevant. This further confirms the high relevance of the term "drama". On the other hand, the genre "artistic" is ignored (the concept "artistic" does not belong to the ontology as this term is undiscriminating: all films can be described as "artistic"). Knowledge fusion is thus based on a qualitative approach of the relationships that exist between the words of the text and the concepts/specific differences of the trade ontology. This makes it possible to extract certainty coefficients pertaining to a film classification based on the contents of the texts written to describe them.

\section{Multimédia}

The previous section illustrated fusion operations carried out with data emanating from a single medium in a successive manner. The fusion of numeric picture data makes it possible to define indicators with enriched semantics, the fusion of textual information permits to enhance the quality of external film descriptors. Moreover, in certain cases, it allows for the validation of coherence between the declared and actual film categories. This section discloses the potential contributions of a mixed media approach while considering solely pictures and texts.

\subsection{Images and Ontology}

This section presents the trade vocabulary through the ontology and the terminology, like the indicators derived from the shot analysis previously presented. Result complementarity is observed. A solution can consist of establishing a supervised classification of images [12] through the management of textual characteristics, a procedure that comprises two components: a learning phase followed by a production step. The learning phase is a five-step process: (a) categorizing a film corpus using the selected terminology, (b) extracting the shot characteristics from this very corpus, (c) creating a space for the image characteristics and placing these extracted characteristics into the corpus, (d) labelling spatial landmarks using terms generated by the textual approach and finally, (e) searching for homogeneous classes [13].
At this level, it is likely that most characteristics will not fit naturally into classes. This can be solved by searching out new characteristics or using adapted classification techniques. In the exploitation step, the classification obtained in the learning phase could be used to categorize other films by the sole exploitation of image data (for films which have very little or no textual description). It is also likely that such a classification supports the textual analysis to confirm certain choices. According to this plan, the selected terminology emanates from the text and the images only reinforce or supplement the text. Of course, the application of this procedure requires a choice of the image descriptors, which are more or less tight in relationship with this terminology. It seems to us that the descriptors attached to the rhythm or the color must be able to be associated with some concepts from the terminology defined on the genres. For example, a film of the drama kind will have an undoubtedly slow rhythm and a dominance of dark colors, whereas a comedy has a more alert rhythm and undoubtedly brighter and clearer colors. Inversely, we can also try to define the visual (audio) characteristics associated to a technique of realization. For example, the use of powder (like coffee, sand...) returns a particular texture and a specific rhythm of images modification. These characteristics are implicitly used by the experts to identify a certain number of realization techniques when they viewed films, but the associations of these characteristics with the other knowledge of the expert are, for the moment, neither explicit, nor are expressed according to the techniques of image analysis.

An alternate approach can consist in establishing an unsupervised classification using the descriptors' joint analysis. The text and images are initially exploited independently so that they can provide their own characteristics. This supposes that the picture analysis manages to deliver characteristics whose semantic nature suffices. To this end, numerical transcriptions/symbolic systems obtained from fuzzy logic can be helpful [14].

After the use of unsupervised classification methods, based on the nearest-neighbour approach, we have to choose a distance. By experiments, we find that the Euclidean distance is the most appropriate for our corpus [15]. Finally, to overcome the difficulties of visualizing and thus analyzing $n$-order data sets (with $n>3$ ) we are using principal component analysis [16], which decorrelates the data. The visualization of the results is performed using only the first three principal components in a 3D plot, as they account for as much of the variability in the data as possible. We obtain clusters: with the help of experts, we have to name and to associate "sense" to these clusters and these factorial components. It provides us with the most suitable clusters that we will seek to name and characterize as finely as possible. In each cluster, we have films that have "close characteristics", characteristics which we will try to express with the assistance of the experts. 


\subsection{Film Shots and Summaries}

As mentioned above, the relationships between film summaries and the ontology concepts or the selected terminology and relationships between the words and indicators provided by the shots can be studied. Many references concerning the dominant colours, the rhythm of the action sequence and the film atmosphere appear in the summary. In the previous example, the following indicators and correlations are obtained: a rather slow rhythm of shot changes at the beginning (wait) which accelerates with the new dramatic actions (confrontation, acceptation), coupled with a high level of activity. Such a rhythm is well-adapted to the story. The selection of colours (medium level of diversity and variety) and their contrasts correlate with the techniques used. However, the warm colours and contrast noted also agree with the summarized history, while emphasizing the enigmatic side of the beginning of film, just like the question marks in the text.

To ensure that images and summaries are complementary, the same qualitative approached is applied, as described above. Moreover, the indicators of actor's presence or indicators about related action and characters (investigated in the image section) would undoubtedly take a preponderant place. Summaries can be an effective tool when searching for these shot elements, much like the elements under the category "film component". Furthermore, the vocabulary and expressions, in summaries, often provide indications as to the atmosphere, information sought by the indicators generated by the images. Finally, the images can help remove potential ambiguities caused by the fact that trade vocabulary is juxtaposed with the extended vocabulary of the text. Although it is still difficult to extract exhaustive relevant knowledge from the texts using the full richness of natural language, various styles, metaphorical expressions or allusions, the suggested approach provides promising avenues, particularly by corroborating "impressions" from the picture and the written texts.

\section{Conclusion}

In this investigation, an authentic application confirms that multimedia data processing is far from being simple and still leaves many unresolved challenges. However, constructive elements for an improved approach using the contents of these multimedia documents are being proposed. On the one hand, text information is used to define a semantic-based terminology and ontology. We have, strong, coherent, reusable specifications for a given and specific field, on which the experts have reached consensus. The ontology and the terminology form the cornerstone of our system. They remove ambiguity in the communication between experts, or between experts and different types of users. They explicitly define the entire trade vocabulary.
Using this ontology, we can exploit some of the richness of natural language found in texts such as film summaries, and use picture data to create indicators to improve the references to the multimedia document content. Bringing together the semantic fields is supplemented by fusion operations which are based on fuzzy logic and a qualitative approach that exploits the image and text components. Finally, adopting a hybrid approach for different media can supplement missing data or increase certainty coefficients in a satisfactory fashion (for example a classification listing the criteria "movie genre", "movie topic", etc.). Although a number of studies are still underway, information and knowledge fusion adds promising complements to formalize and model ontology and terminology based knowledge.

\section{Acknowledgment}

The authors would like to thank CICA [1] and Folimage company [17] for providing us with the animation movies, Ontologos Corp. for providing software (OCW) supporting the OK ontology building.

We also would like to thank D. Memmi for useful comments and suggestions.

\section{References}

[1] Centre International du Cinéma d'Animation "http://www.annecy.org".

[2] Ionescu B., Coquin D., Lambert P., Buzuloiu V., Fuzzy Semantic Action and Color Characterization of Animation Movies in the Video Indexing Task Context, Lecture Notes in Computer Science, Vol. 4398, Eds. S. Marchand-Maillet et al, Springer-Verlag, Berlin Heidelberg, 2007, pp. 119-135.

[3] Beauchene D., Deloule F., Ionescu B., Lambert P., Ontologies et indexations vidéo pour les films d'animation, Atelier Métadonnées et Systèmes d'Information, XXIIIe congrès INFORSID, CD-ROM , Grenoble, mai 2005

[4] Itten, J. The Art of Color: the Subjective Experience and Objective Rational of Color, New York: Reinhold, 1961.

[5] J.P. Desclés, Langages applicatifs, langues naturelles et cognition, Paris, Hermès, 1990.

[6] T. Gruber, Towards principles for the design of ontologies used for knowledge sharing, Int. J. Human Comput. Stud. 43, 1995

[7] C. Roche, Terminologie et ontologie, revue Langage $\mathrm{n}^{\circ} 157$, mars 2005, ed. Larousse. 
[8] http://ontology.univ-savoie.fr/condillac/

[9] M. Lafourcade et V. Prince, Synonymies et vecteurs conceptuels. In proc. of Traitement Automatique du Langage Naturel (TALN'2001), Tours, France, Juillet 2001, pp 233-242

[10] C. Tricot, C. Roche, Visual Information Exploration: A Return on Experience in Knowledge Base Management, Int. Conf. on Artificial Intelligence (IC-AI 2005), Las Vegas, Nevada, USA, June 2005, pp. 955-962.

[11] O. Feiguina, Résumé automatique des commentaires de consommateurs, M.Sc. Université de Montréal, Mai 2006.

[12] C. Burges, A tutorial on Support Vector Machines for Pattern Recognition, DataMining and Knowledge Discovery, Kluwer Acadelic Publishers, vol. 2, n. 2, pp. 12-16,1998.

[13] L. Kaufman, P.J. Rousseeuw, Finding Groups in Data: An Introduction to Cluster Analysis, Wiley, 1990.

[14] Bouchon-Meunier B., Desprès S., Dubois D., Gascuel O., Genoche A., Prade H.,Interface entre symbolique et numérique, Actes des 3ème journées nationales du PRC-IA,Hermès, pp. 89-138, Paris, 1990.

[15] Seber G.A.F, Multivariate Observations, Wiley, New York, 1984.

[16] Jackson J.E., User's Guide to Principal Components, John Wiley and Sons, Inc., 1991.

[17] Folimage: "http://www.folimage.com". 